\title{
Le numérique dans la formation des enseignant-es et du personnel d'encadrement en Afrique
}

\author{
Mona Laroussi, Université de la Manouba, Tunisie
}

\section{DOI : 10.51186/journals/ed.2020.10-2.e350}

\section{Résumé}

Afin de garantir une éducation de qualité, la plupart des pays africains travaillent à renforcer les compétences professionnelles des enseignant-es et personnels d'encadrement. Cela passe entre autres par l'amélioration des méthodes d'enseignement (en/du français dans le respect des langues nationales, des sciences, de la citoyenneté) à la faveur de pratiques innovantes et de nouveaux outils didactiques. Le numérique présente le potentiel qui doit permettre de passer d'une innovation technologique à une innovation pédagogique. Réinventer la formation, c'est une équation qui ne peut être résolue qu'en prenant en compte les contraintes liées aux contextes des apprenant-es et des pays, la massification, l'omniprésence du numérique, les acteurs/trices impliqué-es et les changements dans les rapports aux savoirs. Une des voies pour trouver une solution satisfaisante est d'accepter de faire de l'acte pédagogique une œuvre « ouverte ». Ce modèle impose de repenser la place et le rôle des acteurs et actrices de façon qu'elles/ils puissent faire s'interpénétrer leurs travaux, évoluer et opérer une fertilisation croisée.

Mots-clés : formation des enseignant-es, qualité, numérique éducatif, IFADEM, TESSA

\begin{abstract}
To ensure high-quality education, most African countries are working to strengthen the professional skills of teachers and managerial staff. This includes improving teaching methods (in French, but also in national languages, science and civics) through innovative practices and new teaching tools. Digital solutions can help us turn a technological innovation into a pedagogical one. If teaching is to be reinvented, we must take into account constraints related to the contexts of learners and countries, massification, the ubiquity of digital technology, the stakeholders concerned and changes in our relationship to knowledge. One way to find a satisfactory solution is to make the pedagogical act "open". Using this model requires us to rethink the place and role of the various stakeholders so that they can interact and exchange with each other, allowing a fruitful cross-fertilization to occur.
\end{abstract}

Keywords: teacher training, quality, technology enhanced learning, IFADEM, TESSA 


\section{INTRODUCTION}

Sur le continent africain, l'amélioration de la qualité des enseignements et des apprentissages constitue depuis longtemps et reste aujourd'hui encore une préoccupation essentielle de chacun des États et gouvernements, mais aussi de l'ensemble de la communauté internationale. C'est un enjeu majeur, en témoignent les importants engagements sur la question (par exemple les objectifs de développement durable de I'Unesco dont l'ODD4', la déclaration d'Incheon ${ }^{2}$, les conférences Pace ${ }^{3}$ ou l'agenda à l'échelle de l'Union africaine $e^{4}$ ). Les spécialistes de tous les domaines sont mis à contribution pour analyser certains facteurs devant concourir à une éducation de qualité (Behrens, 2007).

Quels que soient leurs domaines d'expertise et leurs axes d'analyse, tous s'accordent pour penser que cette qualité passe par la maîtrise de la ou des langues d'enseignement, ce qui suppose une maîtrise de cette ou de ces langues par les principaux acteurs/trices que sont les enseignant-es, les premier-es à se trouver au contact des bénéficiaires finales/aux que sont les élèves. Les encadrant-es ${ }^{5}$ et les formateurs/trices de ces enseignant-es se doivent aussi de contribuer à la qualification des enseignant-es par la formation et la production d'outils pédagogiques adéquats. Parmi ces outils, on retrouve par exemple les manuels scolaires, les manuels destinés au suivi pédagogique de proximité et les manuels d'évaluation (sommative et formative).

Les mécanismes de formation des enseignant-es (formation initiale et continue) qui s'appuient, au moins partiellement, sur les orientations curriculaires de chacun des pays, intègrent de plus en plus la question du numérique, tant dans la mise à disposition des ressources aux acteurs et aux actrices que dans les interactions entre ces dernières/iers (relations formateurs/tricesenseignant-es, relations enseignant-es-élèves, relations enseignant-es-encadrant-es pédagogiques).

La présente contribution, relative à l'utilisation du numérique et visant à une comparaison entre un contexte francophone et un contexte anglophone, s'appuie sur des recherches récentes, des expériences de terrain, ainsi que les retours provenant de certains programmes internationaux.

Cet article est écrit du point de vue de la chercheuse que je suis. J'ai en effet mené des études sur des solutions innovantes comme l'enseignement mobile, l'ubiquité, la malléabilité, l'adaptativité ou les profils des apprenant-es (Laroussi, 2020). J'ai toujours travaillé à l'aide de

\footnotetext{
${ }^{1}$ Dans le programme de développement durable, l'objectif 4 stipule : «Assurer l'accès de tous à une éducation de qualité, sur un pied d'égalité, et promouvoir les possibilités d'apprentissage tout au long de la vie ».

2 http://uis.unesco.org/sites/default/files/documents/education-2030-incheon-framework-for-action-

implementation-of-sdg4-2016-fr.pdf

${ }^{3} \mathrm{https}$ ://fr.unesco.org/gem-report/node/2344

${ }^{4}$ https://au.int/sw/node/35416

${ }^{5}$ Le terme d'encadrant est ici préféré à celui d'encadreur, que l'on trouve également dans une partie des études.
} 
méthodes empiriques mêlant observations et tests sur des groupes de taille restreinte, en tentant ensuite d'extrapoler mes constatations pour pouvoir potentiellement les adapter à une population plus large (Cohen, et al., 2017 ; Nieveen \& Folmer, 2013 ; Tricot, et al., 2003).

Parralèlement à cela, j'ai, depuis quelques années, développé une autre approche en privilégiant l'aspect de terrain. Ma pratique sur le terrain est basée sur l'observation, en référence à la participation observante en recherche qualitative (Soulé, 2007). Cette participation observante m'a permis de recueillir des données dans les salles de classes, dans les usages du numérique, dans les courriels transmis ou encore dans les documents rédigés.

II parait évident que l'objectif principal de tout enseignant-e est d'améliorer la qualité de son enseignement. Mon observation était guidée par la question suivante: "Est-ce que la formation dispensée a changé quelque chose dans la façon d'enseigner des personnes formées ? ".

Parallèlement positionnée dans une pratique réflexive de formatrice de formateurs/trices, désirant améliorer ma pratique, cette participation observante s'est transformée en objet de recherche en lien avec la formation des enseignant-es. Au-delà des aspects théoriques sur lesquels repose l'article, il vise à expliciter la situation sur le terrain. En effet, il se trouve parfois qu'une solution très simple peut fonctionner alors que des solutions complexes mobilisant des moyens humains importants échouent.

Après avoir identifié les défis liés à la formation des enseignant-es et les possibles stratégies pour les contourner, je comparerai deux des principaux programmes de formation à distance déployées en Afrique, à savoir IFADEM (Initiative pour la formation à distance des maîtres), francophone, et TESSA (Teacher Education in Sub-Saharan Africa), de fondement anglophone.

\section{LA FORMATION DES ENSEIGNANT-ES}

L'enseignement constitue un métier à part entière, une activité professionnelle en tant que telle: il requiert donc, comme pour toute profession, des compétences de la part des acteurs/trices, que ces derniers/ères peuvent atteindre via une formation adéquate. Cette formation consiste en un ensemble de stratégies et de contenus élaborés sur la base des compétences (les savoirs, savoir-faire, savoir-être) attendues à la fin de l'étape première dite de la formation initiale ; il est également question des prérequis ou bases dont doivent disposer les personnes qui entreprennent cette formation (Yvon \& Saussez, 2010).

La formation des enseignant-es doit en premier lieu répondre à des impératifs d'ordre professionnel et, au-delà des pures connaissances dans les matières enseignées, permettre à ces enseignant-es de maîtriser les stratégies de base nécessaires à leur pratique. Elle doit aussi tenir compte de nouveaux défis, parmi lesquels l'évolution des approches pédagogiques sur la base de nouvelles orientations curriculaires, l'évolution des effectifs des apprenants-es et 
celle des outils pédagogiques ou de tout support permettant aux enseignant-es de mieux exercer leur métier - ou du moins les invitant à l'exercer de manière renouvelée.

Dans la mesure où les systèmes éducatifs sont eux-mêmes en perpétuel changement, le processus de formation des enseignant-es est complexe et se déroule tout au long de leur vie professionnelle, selon différentes modalités parmi lesquelles la formation continue ou l'autoformation.

Au cœur des axes d'amélioration des conditions de formation des enseignant-es se trouve, depuis plusieurs années, l'utilisation du numérique. Or, le numérique est lui-même devenu un domaine de spécialité dans le domaine des sciences de l'éducation. La didactique et la pédagogie (relatives aux enseignant-es, aux élèves ou aux modes d'évaluation par exemple) sont aujourd'hui irriguées, voir dominées, par la prise en compte du numérique. Celui-ci est considéré par beaucoup de spécialistes comme la panacée pour mieux former des enseignantes, pour en former davantage, pour mieux diffuser les outils pédagogiques, pour évaluer plus efficacement et plus finement les performances des élèves comme celles des enseignant-es et proposer en conséquence les systèmes de remédiation les plus adaptés (Soubrié, 2016).

Selon les pays, et en fonction de la demande, le niveau de recrutement des enseignant-es varie fortement. Dans un certain nombre de pays d'Afrique subsaharienne, ce niveau de recrutement se situe à la fin du collège (validé par l'obtention du diplôme de fin d'études du collège, soit le brevet). La durée de la formation, quant à elle, varie d'une année à deux, voire trois. On compte également parmi les élèves-maîtres des titulaires du baccalauréat - la durée de la formation de cette catégorie étant différente de celle des titulaires du brevet. Les deux catégories d'élèves-maîtres enseignent dans les écoles primaires à l'extérieur des grandes villes et n'ont pas toujours la possibilité de suivre des cycles de formation classique dispensés dans des écoles de formation.

L'Institut de statistique de l'Unesco (ISU) évaluait en 2016 les besoins d'enseignant-es du primaire et du secondaire pour la réalisation de l'ODD4 à près de 69 millions de personnes supplémentaires, dont 17 millions pour l'Afrique subsaharienne. De plus, les nécessaires réformes conduiront à recruter de nouveaux profils d'enseignant-es avec de nouveaux besoins de formation. Malgré les efforts des États et des partenaires techniques et financiers en matière de formation, on compte encore un nombre important d'enseignant-es peu, mal ou pas formées. Ainsi, en Afrique subsaharienne, en 2014, seuls environ deux tiers des enseignant-es du pré-primaire et la moitié de ceux du second cycle du secondaire ont suivi une formation (GEMR-UNESCO, 2016).

Au-delà de la forte demande en personnel enseignant, le défi en matière de formation s'inscrit dans la droite ligne du principe fondamental de recherche de la qualité, à savoir l'amélioration des performances des enseignant-es. II est simplement question de voir en elles et eux des professionnel-les du domaine à même de gérer tout type de situation d'enseignement- 
apprentissage pour soutenir les apprenant-es dans le développement de leurs compétences et connaissances.

Cependant, si l'on prend l'exemple des pays d'Afrique subsaharienne, l'important besoin quantitatif en enseignant-es a conduit les responsables des ministères de l'éducation à engager de nouveaux profils d'enseignant-es qui, pour la plupart, ne sont pas passés par des écoles ou des instituts de formation d'enseignant-es idoines. Dans de nombreux pays, l'effectif de ce profil d'enseignant-es n'ayant reçu aucune formation initiale "globale » dépasse le nombre d'enseignant-es « professionnel-les ».

Dans de tels contextes, la formation continue, censée pallier les difficultés rencontrées dans l'exercice du métier, devient désormais le mode de formation initiale. La formation continue devient le mécanisme de certification, le processus pour la titularisation d'une catégorie d'enseignant-es qu'on appelle « contractuel-les » et qui cherchent à intégrer la fonction publique, gage de sécurité de l'emploi.

La formation continue devient de ce fait le mécanisme d'amélioration des performances scolaires mais aussi le levier d'amélioration du savoir-faire du personnel enseignant, contribuant à le motiver et à le légitimer. Bien plus, la participation à un nombre défini de formations continues peut donner droit à un recrutement comme fonctionnaire d'État.

On comprend dès lors les énormes enjeux des dispositifs de formation continue mis en place dans le cadre de programmes nationaux et internationaux. La formation des enseignant-es peut prendre la forme d'une formation initiale ou d'une formation continue - la seconde n'excluant d'ailleurs pas la première. La formation initiale a lieu dans les écoles/centres/instituts de formation des enseignant-es/maîtres. Elle est organisée, en référence au curriculum de chaque pays, autour des quatre axes principaux suivants: (i) les stratégies pédagogiques (techniques d'animation de salles de classe, rédaction de fiches pédagogiques, élaboration et correction des évaluations, etc.); (ii) les connaissances académiques (disciplinaires, pédagogiques, etc.) ; (iii) la capacité à analyser des situations, à réfléchir sa pratique, à développer une attitude réponse des difficultés des apprenants; (iv) les compétences relationnelles - soit la compétence à développer personnellement des connaissances pour faire de l'enseignant-e un-e acteur/trice social-e véhiculant l'appétence à travailler avec ses pairs, la transmission de valeurs et capacités d'adaptation dans une société en perpétuel changement (Plumelle \& Latour, 2012).

Quant à la formation continue, elle concerne les enseignant-es déjà en activité, elle prend en compte les insuffisances constatées au cours des activités de suivi pédagogique, à l'occasion des évaluations des performances de ces enseignant-es. Le contenu ne varie pas fondamentalement de celui de la formation initiale, il prend en compte les insuffisances constatées pour intégrer les remédiations nécessaires. La formation continue peut également prendre la forme d'une formation initiale quand il s'agit d'enseignant-es «contractuel-les » 
recruté-es pour répondre à un besoin pressant, comme c'est très souvent le cas dans plusieurs pays d'Afrique subsaharienne.

\section{LE NUMÉRIQUE DANS LA FORMATION DES ENSEIGNANT-ES}

En ce qui concerne le numérique dans la formation des enseignant-es, autrement dit le « numérique éducatif », c'est un concept qui dépasse la technologie qui le supporte, ouvre de nouvelles voies pour l'éducation, la formation et l'apprentissage. II permet de repenser la structure des actions, usages et pratiques.

Le numérique permet de passer d'une innovation technologique à une innovation pédagogique. II devient ainsi un puissant levier de changement permettant de décloisonner les espaces d'apprentissages (à distance et en présence), de diversifier les styles d'enseignement et les pédagogies déployées (Figure 1) (Bernard \& Fluckiger, 2019).

Le numérique ne transforme pas nécessairement les fondements de la pédagogie ni la manière d'enseigner, mais permet plutôt de l'examiner comme une œuvre « ouverte » : une œuvre dans laquelle les ressources numériques transforment « silencieusement » ou du moins de manière plus ou moins discrète les espaces et les temps d'enseignement et d'apprentissage. Cette transformation silencieuse est concrète en temps de Covid-19, où les espaces et les temps se sont confondus dans un continuum sans limites visibles et nettement définies. Une approche ouverte peut, de ce point de vue, permettre une capitalisation et contribuer, par exemple, à réduire la sensation d'isolement.

Le numérique facilite la différenciation des parcours pédagogiques. Toutefois, le numérique ne peut pas se réduire aux technologies qui permettent uniquement la différenciation du contrôle des élèves. Le numérique doit être étudié à travers les usages. Or, ces usages sont contextualisés et dépendent de différents éléments tels que les profils des apprenant-es et des enseignant-es, les dispositifs utilisés ainsi que les infrastructures associées (Bernard \& Fluckiger, 2019 ; Laroussi, 2020).

Pour instaurer une véritable différenciation pédagogique, il faut mettre en place des scénarios d'apprentissage individualisés, basés ou non sur des dispositifs numériques. Les élèves et les enseignant-es se partagent le contrôle/l'usage à travers des outils qu'elle/ils transforment en instruments de travail, en vue d'atteindre les objectifs pédagogiques définis initialement. 


\section{Figure 1. Styles d'enseignement et pédagogies déployées}

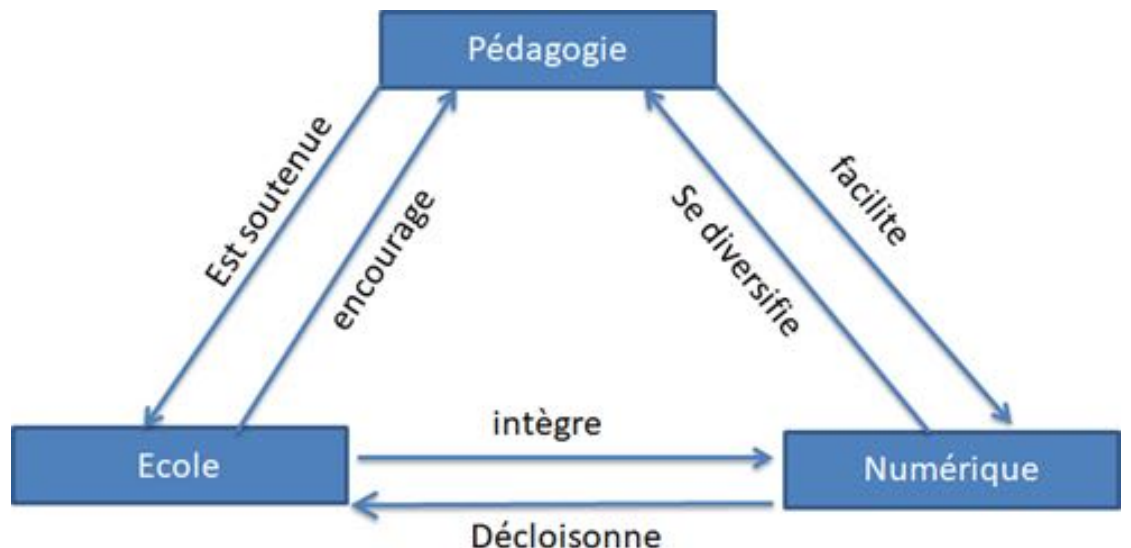

Décloisonne

La crise de la Covid-19 a montré le rôle prépondérant du numérique dans la formation des enseignant-es et pour la formation des enseignant-es.

Les futur-es enseignant-es doivent avoir non seulement la possibilité d'apprendre grâce au numérique mais également les possibilités d'enseigner via le numérique, de transférer leurs expériences d'apprentissage, de faire vivre à leurs élèves et partager avec elles et eux des expériences et des expérimentations intéressantes et enrichissantes.

II est impossible d'isoler l'enseignant-e du contexte dans lequel elle/il exerce, difficile de dissocier le rôle du maître de son élève. Leurs destinées sont en quelque sorte liées. Les élèves d'aujourd'hui, principalement dans les pays développés et les villes, sont généralement considérés comme des "natifs digitaux », qui, de plus en plus, manipulent les ordinateurs et les informations du Web avec facilité et aisance ${ }^{6}$. Le rôle de l'enseignant-e s'est orienté vers un rôle de médiateur/trice entre le savoir et le savoir-faire (Malek \& Laroussi, 2011). L'enseignant-e a compris que son rôle de source unique et absolue d'information dans une salle de classe est révolu et qu'elle/il doit partager ce rôle avec ses élèves et surtout avec Internet.

Le rôle de l'enseignant-e est de guider l'élève dans la recherche d'information et de construire de nouvelles bases de connaissances, tout en exploitant l'interaction sociale avec d'autres apprenant-es pour gagner en efficacité. L'enseignant et l'enseignante devraient aussi pouvoir se positionner comme un passeur et une passeuse de méthodes, un promoteur et une promotrice du discernement et un-e véritable tuteur facilitateur et tutrice facilitatrice. Leur rôle pour aider les élèves à décoder l'information est crucial. Et même quand les adolescent-es parviennent assez aisément à obtenir, récolter des données, leur traitement critique pour les

\footnotetext{
${ }^{6}$ On notera cependant que, par exemple en France, la crise sanitaire du printemps 2020 et le confinement ont révélé de réels problèmes sur certains territoires ou dans certaines familles où l'accès aux ressources numériques est limité ou très partagé, inégal en tout état de cause : une fratrie nombreuse se partageant un unique accès à Internet, ou encore des collégien-nes ne disposant que d'un téléphone portable au forfait limité pour suivre des cours en ligne.
} 
transformer en une information de qualité utilisable reste problématique et nécessite un accompagnement attentif (Malek, et al., 2019).

L'aisance informatique n'est de toute façon pas le cas dans la plupart des pays africains, où les élèves ne sont pas toutes et tous des « natifs/ves digitales/aux ». Elles/ils ne peuvent pas l'être par manque de moyens. De plus, l'enseignant-e se retrouve très souvent dans une situation de diversité des tâches, une diversité d'élèves et en manque criant de moyens.

Le numérique éducatif est présenté comme pouvant rendre accessible au plus grand nombre l'éducation, et notamment les ressources d'apprentissage. C'est, en tant que tel, un outil pertinent de massification de l'enseignement. L'absolue nécessité de former un nombre très important d'enseignant-es dans des délais très courts a déjà été évoquée. Prendre en charge ce grand nombre conduit à innover, à trouver des moyens adaptés pour élaborer un nouveau référentiel de compétences tenant compte du numérique, à différencier la pédagogie pour prendre en compte l'hétérogénéité, à intégrer dans la formation les moyens et les dispositifs permettant de rechercher l'équité. Cependant, les obstacles pour y parvenir sont encore très nombreux dans les pays en développement (Depover, et al., 2007).

\section{CADRE THÉORIQUE}

La formation des enseignant-es par le numérique est un projet complexe qui ne saurait en aucun cas se réduire à la simple substitution d'un support imprimé par un support numérique. La réelle intégration du numérique dans un dispositif de formation se présente avant tout comme une innovation technologique complexe, de plusieurs points de vue, et qui introduit d'importants changements dans les processus d'enseignement et d'apprentissage, tout particulièrement dans ceux qui relèvent de la médiatisation et de la médiation (Peraya, 2006).

La médiatisation relève des processus d'ingénierie pédagogique, tandis que la médiation porte sur l'analyse de l'impact de «l'instrument », tel que le définissent Rabardel (1995) et Rabardel et Samurçay (2001) sur l'activité et les comportements humains (Charlier, et al., 2006). En effet, pratiquement tous les spécialistes des questions concernant l'utilisation des Technologies de l'information et de la communication dans l'enseignement (TICE) ou la formation et l'apprentissage, s'accordent pour penser que l'utilisation du numérique dans la formation comporte une importante dimension humaine qu'il convient de prendre en compte en plus de la dimension technologiques. Et cela est d'autant plus vrai dans la formation hybride (Peraya, 2008).

Cette dimension humaine concerne l'ensemble de la chaine des acteurs et actrices (concepteurs/trices de cours, tuteurs/trices, apprenant-es, etc.) engagés dans le processus de formation. La maîtrise du paramètre humain permet de définir les conditions adéquates pour atteindre les objectifs fixés par le processus. C'est un facteur décisif de réduction et de prévention des risques d'échec et une garantie de bonne conformité avec les critères d'utilité, 
d'utilisabilité et d'acceptabilité qui fondent l'efficacité d'un dispositif techno-pédagogique de formation hybride utilisant le numérique (Tricot, et al., 2003).

Ces différents rôles impliquent la maîtrise d'un ensemble de compétences nécessaires pour la réussite des apprentissages. Les conditions dans lesquelles un-e apprenant-e (dans notre cas un-e enseignant-e) arrive dans le dispositif ne sont donc pas à négliger. En fonction de son passé, et pas uniquement d'apprenant-e dans un cadre académique, il/elle peut plus ou moins être déstabilisé-e dans ses habitudes et ses représentations des situations de formation, notamment par l'isolement, la nécessité de surmonter les obstacles d'ordre technique, la gestion de l'autonomie, etc. (Deschryver \& Peraya, 2003). Une capacité fondamentale pour réussir dans sa fonction d'apprenant-e est celle d'être " capable de se prendre en charge et de développer» ce que Linard (2001) appelle « la capacité de distanciation cognitive » (p. 2).

Cette capacité d'autonomie dépend de plusieurs facteurs, dont le niveau de maîtrise de la technologie avec laquelle l'apprenant-e arrive dans le dispositif. Tout cela implique que dans le projet envisagé par IFADEM, replacer le contexe, la connaissance des prérequis de la part de l'apprenant-e en matière de numérique, se pose comme une condition essentielle de réussite. Ces prérequis sont de divers ordres : matériel, pratique, psychologique et socioculturel. II s'agit de tout ce dont ces enseignant-es devraient disposer concernant les TIC et les technologies connexes avant d'entrer en formation - soit, les technologies qui leur sont accessibles, les technologies utilisées, les usages établis et les représentations construites.

\section{DEUX EXEMPLES DE PROGRAMMES DE FORMATION IFADEM/TESSA}

Plusieurs programmes ont cherché à relever les défis de la formation des enseignant-es et du personnel enseignant en Afrique subsaharienne. II s'agit notamment de l'Initiative pour la formation à distance des maîtres (IFADEM), présentée ici tel que déployée au Sénégal et du projet Teacher Education in Sub-Saharan Africa (TESSA).

\subsection{IFADEM}

IFADEM est mise en place par l'Agence universitaire de la Francophonie (AUF) et l'Organisation internationale de la Francophonie (OIF) en co-construction avec les différents États ${ }^{7}$ (2006). L'initiative s'appuie sur un dispositif de formation continue qui associe la formation à distance et la formation présentielle, les ressources écrites et audio, le tutorat de proximité et une initiation au numérique (informatique/bureautique et Internet). Ce modèle permet de renforcer les compétences des maîtres déjà en poste tout en ne les contraignant pas à quitter la salle de classe (Baron \& Villemonteix, 2016).

\footnotetext{
${ }^{7}$ La commande politique de I'IFADEM émane de la déclaration du Xlème Sommet des chefs d'État et de gouvernement ayant le français en partage (Bucarest, 2006).
} 
L'objectif global concerne le renforcement des capacités professionnelles des enseignant-es, principalement du primaire. II s'agit de contribuer à l'amélioration de la qualité de l'éducation de base dans le cadre de la stratégie de l'Éducation pour tous dans les pays francophones, par le renforcement des compétences professionnelles. Cet objectif global se décline en trois objectifs spécifiques : i) mettre en œuvre des dispositifs de formation à distance ou partiellement à distance; ii) améliorer les méthodes d'enseignement par des pratiques innovantes, l'emploi de nouveaux outils et méthodes pédagogiques ; iii) appuyer la définition de stratégies nationales de formation ${ }^{8}$.

Le principe d'action prioritaire d'IFADEM réside dans le caractère ascendant de la démarche, en termes tant de gouvernance et de processus que de contenus. Le projet repose sur une approche de co-construction avec les pays, qui intègre ainsi des initiatives dans et à leurs contextes administratifs locaux. II assure la formation d'acteurs locaux et d'actrices locales qui participent sur le terrain au développement et à la mise en œuvre du dispositif. II s'agit notamment de rédacteurs/trices de contenus pédagogiques, de supports de formation et d'animation.

Le Sénégal, qui bénéficie d'une expérience de formation à distance depuis plusieurs décennies, a inscrit l'utilisation des technologies dans tous ses documents relatifs à la politique éducative en vigueur. Ce contexte, associé à une bonne couverture du pays en ressources, en électricité et en réseau Internet, a permis de concevoir la phase expérimentale d'IFADEM en dotant chaque enseignant-e bénéficiaire d'une tablette tactile. La tablette lui donne ainsi accès à une plateforme de formation en ligne accueillant les livrets ${ }^{9}$ et les ressources complémentaires, et favorisant les échanges entre pairs et avec les encadrant-es. On notera en passant que les tablettes peuvent être remplacées par des téléphones intelligents - à Madagascar, l'expérience de cet usage a été réalisée avec succès, par exemple ${ }^{10}$.

Les objectifs définis pour IFADEM au Sénégal étaient de quatre ordres : i) améliorer les compétences professionnelles des enseignant-es du primaire déjà en poste en prenant en compte leurs besoins de formation et leur environnement de travail et de vie ; ii) contribuer à la modernisation des méthodes pédagogiques, des modes d'accès et d'appropriation des savoirs et des savoir-faire nécessaires par l'autoformation assistée par des tuteurs/trices et en recourant aussi aux TIC ; iii) promouvoir un enseignement de qualité dans un milieu multilingue ; et, iv) contribuer à la promotion des instituteurs/trices adjoint-es au grade d'instituteur/trice ordinaire.

L'efficacité du programme a été confirmée par la réussite à 85\% aux épreuves de certification des instituteurs/trices adjoint-es formé-es, qui sans nul doute doit beaucoup aux nouvelles connaissances et compétences acquises par l'exploitation des livrets pédagogiques. Ces

\footnotetext{
${ }^{8}$ https://www.francophonie.org/sites/default/files/2019-10/Evaluation-ifadem\%202010.pdf

${ }^{9}$ Un livret est un support de formation qui vise à développer les compétences linguistiques et didactiques des enseignant-es.

10 https://ifadem.org/sites/default/files/divers/eval_ifadem_mada_final_26fev2014_der.pdf
} 
livrets avaient en effet cherché à prendre en compte les difficultés d'enseignement et d'apprentissage selon les niveaux définis dans les programmes officiels du primaire. Grâce à I'utilisation des TIC, l'aspect « à distance » constitué par le travail individuel sur les livrets a été mieux mis en œuvre que dans d'autres pays (dans lesquels la plupart des stagiaires se contentent du travail avec les tuteurs/trices lors des regroupements).

\subsection{TESSA}

De son côté, l'Open University du Royaume Uni a lancé le programme TESSA, en 2005, pour lutter contre le manque de formation des enseignant-es du primaire en Afrique subsaharienne. C'est un projet de recherche-action qui, dans son développement en tant que coopération internationale, fait intervenir une douzaine d'institutions internationales et nationales (tessafrica.net/fr).

TESSA comporte deux axes majeurs, à savoir, d'une part, la conception et l'amélioration des dispositifs de formation des enseignant-es et, d'autre part, la mise à disposition, pour les enseignant-es, de ressources éducatives libres modulables et exploitables dans leurs contextes pédagogiques. En effet, le portail TESSA permet aux enseignant-es d'accéder à un grand nombre de ressources éducatives libres qui leur permettent de renforcer leurs capacités. Ces ressources, qui couvrent cinq domaines de formation (compétences de la vie courante, alphabétisation, mathématiques, sciences, sciences humaines et arts) et qui sont disponibles en quatre langues (anglais, arabe, français et swahili), sont conçues par des expert-es qui ont aussi élaboré le curriculum qui les accompagne. Ces ressources permettent une autoformation individualisée et sont par ailleurs proposées selon un principe d'unités d'étude, ce qui permet de les adapter au niveau et aux besoins d'une classe donnée. Leurs concepteurs et conceptrices ont bien entendu tenu compte des nécessités des curriculums spécifiques pour les élaborer. De plus, une aide pratique est disponible, sous forme de guides d'utilisation : un guide pour les enseignant-es/formateurs/trices et l'autre pour les formateurs/trices de formateurs/trices.

Le portail Tessa se veut interactif et œuvre dans un esprit de communauté : il « abrite aussi un forum d'échanges d'idées sur la création et le partage de ressources pédagogiques. Un accent particulier est mis sur le partage via l'espace PartaTESSA qui permet aux enseignant-es d'explorer, de partager, d'adapter et d'ajouter leurs ressources propres pour l'éducation et la formation » (Depover, et al., 2016, p. 210).

IFADEM et TESSA présentent d'évidentes similitudes dans leurs objectifs: les deux programmes visent à renforcer les compétences des enseignant-es et à développer les ressources pédagogiques. Le multilinguisme structurel de TESSA constitue une particularité innovante de ce programme, et la gouvernance d'IFADEM (OIF-AUF-pays concerné) lui confère une place à part dans les initiatives déployées sur le continent africain. II faut néanmoins relever qu'IFADEM se concentre sur l'enseignement du français d'une part et sur l'initiation des enseignant-es à l'utilisation du numérique éducatif d'autre part. 


\section{CONCLUSION}

La formation des enseignantes et des enseignants du primaire et du cycle fondamental présente beaucoup d'insuffisances qui ont un impact négatif sur la qualité des maîtres en poste. Au vu des prestations et des résultats d'apprentissages dans de nombreux pays, il est impératif et urgent d'améliorer la formation des enseignant-es (Ouedraogo, 2011). L'inadéquation entre la formation que les enseignant-es reçoivent et les compétences exigées par l'exercice de leur fonction semble constituer un des facteurs majeurs de cette situation. En effet, l'enseignant-e reste l'acteur/trice essentiel-le des apprentissages et de la formation dans un système éducatif normé. L'Afrique subsaharienne a besoin d'enseignant-es de qualité pour atteindre ses objectifs de développement économique et social. Dans cette perspective, la formation initiale et continue des enseignant-es constitue une condition de base et la plus grande attention doit lui être portée.

II faut aussi penser à former les cadres institutionnel-les dans les ministères qui décident des formations des enseignant-es et qui mettent en place les politiques sectorielles de l'éducation pour qu'elles et ils intègrent les formations, le numérique et l'éducation ouverte dans leurs stratégies. Le personnel intermédiaire n'est pas non plus à négliger : c'est chaque maillon de la chaîne qu'il convient de renforcer.

Bien qu'encore inégale, la formation continue des enseignant-es en Afrique subsaharienne semble sur la bonne voie, avec des activités nationales soutenues par des coopérations bilatérales et multilatérales. De ce point de vue, l'usage du numérique éducatif est crucial et son apport indispensable dans des situations de crise comme celle qu'a provoquée la pandémie liée à la Covid-19. Le constat est unanime sur le rôle indispensable joué par les enseignant-es dans la continuité des activités et la solidité des systèmes éducatifs.

En tout état de cause, quels que soient les progrès réalisés dans le domaine de la didactique et de la pédagogie, de la formation initiale et continue, il reste indispensable de prendre en compte les infrastructures et le taux de la couverture numérique dans les pays concernés par l'éducation digitale, en particulier dans les zones où évoluent les enseignant-es : avant même la pédagogie, l'électricité et l'accès à l'Internet se trouvent structurellement au cœur de la problématique et de la réflexion à mener. Les observations menées sur le terrain montrent que la fracture numérique à l'intérieur des pays et entre les pays ne cesse de se creuser et que les usages dépendent très souvent des infrastructures.

\section{RÉFÉRENCES}

Baron, G. L., \& Villemonteix, F. (2016). Accompagnement et supervision des maîtres du primaire. Réflexions issues du projet de recherche SUPERE-RCF. In C. Depover, P.-Y. Dieng, S. Gasse, J.-F. Maynier, \& J. Wallet (Eds). Repenser la formation continue des 
enseignants en Francophonie : I'initiative Ifadem (pp. 97-108). Paris : Éditions des archives contemporaines.

Behrens, M. (2007). Qualité en éducation : Pour réfléchir à la formation de demain. Québec, QC : Presse de l'Université du Québec.

Bernard, F., \& Fluckiger, C. (2019). Innovation technologique, innovation pédagogique. SpiraleRevue de recherches en éducation, 1, 3-10.

Charlier, B., Deschryver, N., \& Peraya, D. (2006). Apprendre en présence et à distance. Distances et savoirs, 4(4), 469-496.

Cohen, L., Manion, L., \& Morrison, K. (2017). Research methods in education. New York, NY: Routledge.

Depover, C., Karsenti, T., \& Komis, V. (2007). Enseigner avec les technologies : favoriser les apprentissages, développer des compétences. Québec, QC : Presse de I'Université du Québec.

Depover, C., Dieng, P. Y., Gasse, S., Maynieret, J.-F., \& Wallet, J. (2016). Repenser la formation continue des enseignants en Francophonie : I'initiative IFADEM. Paris : Éditions des archives contemporaines.

GEMR-UNESCO. (2016). Global Education Monitoring Report: Education for People and Planet. Paris: UNESCO.

https://fr.unesco.org/gem-report/node/1279

Laroussi, M. (2011). E-learning 2.0 virage ou mirage. In M. Ben Henda, \& E. Tonyé (Eds). TIC et éducation en Afrique : applications, recherche et perspectives (pp. 57-70). Paris : L'Harmattan.

Laroussi, M. (2020). Adaptativité et contextualisation dans l'ingénierie et la réingénierie des Environnements Informatiques de l'Apprentissage Humain. Habilitation à diriger les recherches. Tunis : École nationale des sciences informatique Tunis.

Linard, M. (2001). L'autonomie de l'apprenant et les TIC. http://oav.univpoitiers.fr/rhrt/2000/table_ronde_3.htm

Malek, J., \& Laroussi, M. (2011). A model-driven approach for modelling and simulating new malleable learning scenarios for digital native generations. International Journal of Learning Technology, 6(3), 288-319.

Malek, J., Laroussi, M., Ghezala, H. B., \& Nefzi, M. (2019). A methodology for designing mobile, pervasive, seamless and loT-based learning scenarios: language, process and supporting tools. International Journal of Mobile Learning and Organisation, 13(3), 255-285.

Nieveen, N., \& Folmer, E. (2013). Formative evaluation in educational design research. Design Research, 153, 152-169.

Ouedraogo, R. M. (2011). Principes du développement et de la formation des enseignants Stratégies pour l'amélioration des conditions de travail des enseignants des enseignants et leur rétention dans les écoles en Afrique. Addis Abeba : UNESCO.

Deschryver, N., \& Peraya, D. (2003). STAF17 - Réalisation d'un dispositif de formation entièrement ou partiellement à distance. La formation à distance, un cadre de référence. https://tecfa.unige.ch/tecfa/teaching/FFL/Textes/Textes_obligatoires/miel_.dipositif_fepad. pdf 
Peraya, D. (2008). Un regard critique sur les concepts de médiatisation et médiation : nouvelles pratiques, nouvelle modélisation. Les Enjeux de l'information et de la communication. Les Enjeux de l'information et de la communication. https://archiveouverte.unige.ch/unige:17665

Plumelle, B., \& Latour, M. (2012). La Formation initiale et continue des enseignants : Allemagne, Angleterre, Finlande. Sèvres: Centre de ressources et d'ingénierie documentaires. https://www.france-educationinternational.fr/sites/default/files/migration/dossierdoc/docs/formation-initiale-et-continueenseignants.pdf

Rabardel, P. (1995). Qu'est-ce qu'un instrument ? Les dossiers de l'Ingénierie éducative, 19, 61-65.

Rabardel, P., \& Samurcay, R. (2001, March 21-23). From artifact to instrument-mediated learning. Symposium on New challenges to research on Learning, Helsinki.

Soubrié, T. (2016). La pratique réflexive, une dimension centrale dans la formation des enseignants au numérique. Numérique et formation des enseignants de langue. In C. Olliver, T. Gaillat, \& L. Puren (Eds). Numérique et formation des enseignants de langue (pp.1-18). Paris : Pistes et imaginaires, Éditions des archives contemporaines.

Soulé, B. (2007). Observation participante ou participation observante? Usages et justifications de la notion de participation observante en sciences sociales. Recherches qualitatives, 27(1), 127-140.

Tricot, A., Plégat-Soutjis, F., Camps, J. F., Amiel, A., Lutz, G., \& Morcillo, A. (2003). Utilité, utilisabilité, acceptabilité : interpréter les relations entre trois dimensions de l'évaluation des ElAH. Environnements Informatiques pour l'Apprentissage Humain, 36, 391-402. https://edutice.archives-ouvertes.fr/edutice-00000154

Yvon, F., \& Saussez, F. (2010). Analyser l'activité enseignante des outils méthodologiques et théoriques pour l'intervention et la formation. Québec : Presses de l'Université Laval.

Open Access Publications - Bibliothèque de l'Université de Genève Creative Commons Licence 4.0

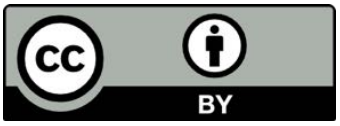

